PROCEEDINGS OF THE

AMERICAN MATHEMATICAL SOCIETY

Volume 127, Number 12, Pages 3711-3719

S 0002-9939(99)04920-5

Article electronically published on May 6, 1999

\title{
CONSTRUCTIVE MODELS OF UNCOUNTABLY CATEGORICAL THEORIES
}

\author{
BERNHARD HERWIG, STEFFEN LEMPP, AND MARTIN ZIEGLER
}

(Communicated by Carl G. Jockusch, Jr.)

\begin{abstract}
We construct a strongly minimal (and thus uncountably categorical) but not totally categorical theory in a finite language of binary predicates whose only constructive (or recursive) model is the prime model.
\end{abstract}

\section{Introduction}

Effective (or recursive) model theory studies the degree to which constructions in model theory and algebra can be made effective. A presentation of a countable model $\mathcal{M}$ is an isomorphic copy $\mathcal{N}$ with universe $N=\omega$. An effective (or computable, or recursive) presentation is one where all the relations, functions, and constants on $\mathcal{N}$ are given by uniformly computable functions. Now, for a countable model $\mathcal{M}$ of a first-order theory $T$, there are various degrees to which the construction of $\mathcal{M}$ can be made effective: We call the model $\mathcal{M}$ constructive (or recursive, or computable) if it has an effective presentation, or equivalently if its open diagram (i.e., the collection of all quantifier-free sentences true in $(\mathcal{M}, a)_{a \in M}$ (in some presentation) is computable (or recursive)). We call the model $\mathcal{M}$ decidable if its elementary diagram (i.e., the collection of all first-order sentences true in $(\mathcal{M}, a)_{a \in M}$, in some presentation) is decidable (i.e., computable). Obviously, any decidable model is constructive, but the converse fails. In fact, the study of constructive models is much harder than the study of decidable models since, in the former case, much less is known about the first-order theory.

Effective model theory has been particularly active in cases where the first-order theory has few countable models. A special case here, which is well-understood classically (i.e., without regard to effectiveness), is that of uncountably categorical theories. Such theories abound in algebra and model theory (e.g., algebraically closed fields, vector spaces, etc.) and were in fact the starting point of modern model theory with Morley's famous categoricity theorem [Mo65]. By a classical theorem of Baldwin and Lachlan [BL71], the countable models of an uncountably

Received by the editors October 20, 1997 and, in revised form, February 20, 1998.

1991 Mathematics Subject Classification. Primary 03C57, 03D45.

Key words and phrases. Constructive/recursive/computable model, uncountably categorical first-order theory, strongly minimal set, unsolvable word problem, Cayley graph.

The first author was supported by a grant of the British Engineering and Physical Sciences Research Council (Research Grant no. GR/K60503).

The second author's research was partially supported by NSF grant DMS-9504474 and a grant of the British Engineering and Physical Sciences Research Council (Research Grant no. GR/K60497). 
but not totally categorical theory $T$ form an elementary chain $\left\{\mathcal{M}_{\kappa}\right\}_{\kappa \leq \omega}$. (Here, $\mathcal{M}_{0}$ is the prime model, and $\mathcal{M}_{\omega}$ is the countably saturated model of $T$.) By a theorem of Harrington [Ha74] and Khisamiev (or Hisamiev) [Hi74], any countable model of a decidable uncountably categorical theory is decidable. The situation for constructive models of uncountably (but not totally) categorical theories $T$ is much more difficult: The fact that some countable models of $T$ are constructive does not imply that all are. In order to show how complicated things can become here, let us define the spectrum of constructive models of $T$ by

$$
\operatorname{SCM}(T)=\left\{\kappa \leq \omega \mid \mathcal{M}_{\kappa} \text { is constructive }\right\} .
$$

The following subsets of $\omega+1$ can be realized as a spectra of constructive models:

(1) easy: $\operatorname{SCM}(T)=\omega+1$.

(2) Gončarov [Go78]: $\operatorname{SCM}(T)=\{0\}$.

(3) Kudaŭbergenov [Ku80]: $S C M(T)=\{0,1, \ldots, n\}$ for arbitrary $n \in \omega$.

(4) Khoussainov, Nies, Shore [KNSta]: $S C M(T)=\omega$.

(5) Khoussainov, Nies, Shore [KNSta]: $S C M(T)=\{1,2, \ldots, \omega\}$.

It is unknown exactly which subsets of $\omega+1$ can be realized as spectra of constructive models (see the end of this paper for further comments).

All the above-mentioned results (except (1)) use infinite languages. The question arises as to whether similar results can also be achieved for finite languages. The main result of this paper is to give a first affirmative answer, namely, an analogue of Gončarov's result for a finite language of binary predicates. (Note that any uncountably categorical theory in a finite language of unary predicates is actually totally categorical.)

\section{Strongly minimal theORIES in A BinARY LANGUAGE}

We begin with a definition.

Definition. Let $\mathcal{L}=\left\{R_{1}, \ldots, R_{k}\right\}$ be a finite relational binary language and $\mathcal{M}$ an $\mathcal{L}$-structure. $\mathcal{M}$ carries in a natural sense the structure of a graph: there is an edge between two distinct points $a$ and $b$ if there exists $i$ such that $R_{i} a b$ or $R_{i} b a$ holds. We freely use graph-theoretic notions and refer thereby to this graph. E.g., we say $\mathcal{M}$ has finite valence if every point has only finitely many neighbors in the graph. The distance $d(a, b)$ between $a, b \in \mathcal{M}$ is the length of the shortest path connecting the points. For $a \in \mathcal{M}, B_{i}(a)$ is the set $\{c \in \mathcal{M} \mid d(a, c) \leq i\}$ and the connected component of $a$ is the set $B(a)=\bigcup_{i \in \omega} B_{i}(a)$.

The following lemma is partially contained in Ivanov [Iv89], [Iv89b]:

Lemma. Let $\mathcal{L}$ be a finite language consisting of binary relation symbols. Let $\mathcal{M}$ be an infinite $\mathcal{L}$-structure of finite valence.

a) $\mathcal{M}$ is strongly minimal iff for every $i \in \omega$ there exist a finite $\mathcal{L}$-structure $\mathcal{C}_{i}$ and $c_{i} \in \mathcal{C}_{i}$ such that for almost all $a \in \mathcal{M},\left(B_{i}(a), a\right) \cong\left(\mathcal{C}_{i}, c_{i}\right)$.

b) If $\mathcal{M}$ is strongly minimal and the structures $\mathcal{C}_{i}$ are as in a), then there exists up to isomorphism a unique connected $\mathcal{L}$-structure $\mathcal{C}$ such that, for every $d \in \mathcal{C}$ and $i \in \omega,\left(B_{i}(d), d\right) \cong\left(\mathcal{C}_{i}, c_{i}\right)$.

c) If $\mathcal{M}$ is strongly minimal and $\mathcal{C}$ is as in b), then every elementary extension of $\mathcal{M}$ is a disjoint union of $\mathcal{M}$ and copies of $\mathcal{C}$. 
Proof. Suppose first that $\mathcal{M}$ is strongly minimal and fix $i \in \omega$. For $a \in \mathcal{M}$ the isomorphism type of $\left(B_{i}(a), a\right)$ is a first-order property of $a$. As there are only finitely many such isomorphism types, there must be a structure $\left(\mathcal{C}_{i}, c_{i}\right)$ such that, for infinitely many $a \in \mathcal{M},\left(B_{i}(a), a\right) \cong\left(\mathcal{C}_{i}, c_{i}\right)$. By strong minimality this must hold for almost all $a \in \mathcal{M}$.

Now let us suppose that the right hand side of a) holds. On the way to proving that $\mathcal{M}$ is strongly minimal, we will prove b) and c). For every $i$ there exists an embedding from $\left(\mathcal{C}_{i}, c_{i}\right)$ into $\left(\mathcal{C}_{i+1}, c_{i+1}\right)$. Thus we can suppose that $\mathcal{C}_{i} \subseteq \mathcal{C}_{i+1}$ and $c_{i}=c_{i+1}$. Let $\mathcal{C}=\bigcup_{i \in \omega} \mathcal{C}_{i}$ and $c=c_{i} \in \mathcal{C}$. Then for every $i \in \omega$ we have $\left(B_{i}(c), c\right) \cong\left(\mathcal{C}_{i}, c\right)$. In fact $(C, c)$ is uniquely determined by this property: If $\mathcal{D}$ is a connected structure and $d \in \mathcal{D}$ satisfies for every $i \in \omega:\left(B_{i}(d), d\right) \cong\left(\mathcal{C}_{i}, c_{i}\right)$, then $(\mathcal{D}, d) \cong(\mathcal{C}, c)$. To see this, look at the set $\left\{p \mid \exists i \in \omega\right.$ such that $p:\left(B_{i}(d), d\right) \cong$ $\left.\left(B_{i}(c), c\right)\right\}$ ordered by extension. This set is a finitely branching tree of infinite height, and by König's Lemma it has an infinite branch in it, which gives rise to an isomorphism $(\mathcal{D}, d) \cong(\mathcal{C}, c)$. This proves uniqueness in $\mathrm{b})$.

Now let us take an elementary extension $\mathcal{M}_{1}$ of $\mathcal{M}$. Let $i \in \omega$. As there are only finitely many exceptional points $b$ in $\mathcal{M}$ not satisfying $\left(B_{i}(b), b\right) \cong\left(\mathcal{C}_{i}, c\right)$, the same number of points, and in fact the same points, are exceptional in $\mathcal{M}_{1}$ in this sense. This means that for every $a \in \mathcal{M}_{1}-\mathcal{M},\left(B_{i}(a), a\right) \cong\left(\mathcal{C}_{i}, c\right)$, which implies that $(B(a), a) \cong(\mathcal{C}, c)$. As all the points in $B(a)$ are also in $\mathcal{M}_{1}-\mathcal{M}$, we have proved that for every $d \in B(a)$ and therefore also for every $d \in \mathcal{C}:\left(B_{i}(d), d\right) \cong\left(\mathcal{C}_{i}, c\right)$, which finishes the proof of b) and c).

Furthermore, for every $a, b \in \mathcal{M}_{1}-\mathcal{M}$ there is an automorphism fixing $\mathcal{M}$ and mapping $a$ to $b$. Thus all the points in $\mathcal{M}_{1}-\mathcal{M}$ have the same type over $\mathcal{M}$, which means that there exists only one nonalgebraic type over $\mathcal{M}$. This implies that $\mathcal{M}$ is strongly minimal.

From the above lemma it follows that if $\mathcal{M}$ is as above, and if $a_{1}, \ldots, a_{n}$ are elements in $\mathcal{M}$, then the algebraic closure of these elements is $\operatorname{acl}(\emptyset) \cup B\left(a_{1}\right) \cup \ldots \cup$ $B\left(a_{n}\right)$. Thus the geometry of the strongly minimal set $\mathcal{M}$ is disintegrated. In fact, the assumption that $\mathcal{M}$ has finite valence is not essential: Let $\mathcal{L}=\left\{R_{1}, \ldots, R_{n}\right\}$ be the binary language. Let $x, y$ be a generic pair of elements. For every $R_{i}$, if $x R_{i} y$ holds, we replace $R_{i}$ by $\neg R_{i}$. So we can assume that, for generic $x, y, \neg x R_{i} y$ holds. This means by strong minimality that for generic $x$ there exists only finitely many $y$ such that $x R_{i} y$ holds. Therefore there are only finitely many points in $\mathcal{M}$, which have infinitely many neighbors and in fact we can assume there are no such points, i.e. we can assume that $\mathcal{M}$ has finite valence. In fact a similar argument works also in the case of an infinite language. So we have the following proposition.

Proposition. Let $T$ be a strongly minimal theory in a binary relational language. Then the geometry of the theory is disintegrated.

\section{THE THEOREM AND PROOF}

The following theorem is the main result of this paper.

Theorem. There is a first-order theory $T$ in a language $\mathcal{L}$ of three binary relation symbols such that

(i) $T$ is an uncountably but not totally categorical theory;

(ii) the prime model of $T$ is constructive; and 
(iii) all the other countable models of $T$ are not constructive; in fact, given any noncomputable $\Delta_{2}^{0}$-degree $\mathbf{a}$, there is a theory $T$ such that the nonprime models have presentations exactly of any degree $\geq \mathbf{a}$.

Moreover, $T$ is strongly minimal.

Proof. We construct a prime model $\mathcal{M}_{0}$ in a language $\mathcal{L}=\left\{R_{0}, R_{1}, R_{2}\right\}$ of three binary relation symbols and show that $T=\operatorname{Th}\left(\mathcal{M}_{0}\right)$ and $\mathcal{L}$ satisfy the claims of our theorem. The prime model will consist of the disjoint union of an infinite number of finite so-called Cayley graphs; the other countable models $\mathcal{M}_{\kappa}$ will then consist of $\mathcal{M}_{0}$ plus the disjoint union of $\kappa$ many copies of a fixed infinite Cayley graph. The main idea is that the word problem in the finite groups corresponding to the finite Cayley graphs will be uniformly computable, while the word problem in the infinite group corresponding to the infinite Cayley graph will be unsolvable.

To this end, we first establish a group-theoretical lemma.

Lemma. Let $F$ be a free group of rank 3 (generated by $g_{0}, g_{1}$, and $g_{2}$, say). Then for every $\Delta_{2}^{0}$-degree a there is a sequence $\left\{N_{k}\right\}_{k \in \omega}$ of subgroups of $F$ such that

(i) $\left\{N_{k}\right\}_{k \in \omega}$ is a uniformly computable sequence of normal subgroups of finite index in $F$, so $F / N_{k}$ has a uniformly solvable word problem;

(ii) for each $w \in F$, the set $\left\{k \in \omega \mid w \in N_{k}\right\}$ is either finite or cofinite, so that the pointwise limit $N=\left\{w \in F \mid\left\{k \in \omega \mid w \in N_{k}\right\}\right.$ is cofinite $\}$ of the $N_{k}$ exists and is again a normal subgroup of $F$; and

(iii) $N$ has Turing degree $\mathbf{a}$.

Proof of the lemma. Fix a $\Delta_{0}^{2}$-subset $A$ of natural numbers that belongs to a and assume that $0 \in A$. Also fix an effective sequence $\left(A_{s}\right)_{s \in \omega}$ of finite sets of natural numbers, such that $A$ is the pointwise limit of the $A_{s}$. Assume further that for every $s, A_{s}$ is contained in $[0, s]$ and $0 \in A_{s}$.

For the following we need two notions from group theory, the definition of which we include (see, e.g., Rotman [Ro95]):

Definition. 1. If $A$ and $B$ are groups and $\varphi: B \rightarrow \operatorname{Aut}(A)$ is a group automorphism (that is, $B$ acts on $A$ ), we define the semidirect product of $A$ and $B$ (which also depends on $\varphi$ ) to be the set $A \times B$ equipped with the group operation

$$
\left(a_{1}, b_{1}\right) \cdot\left(a_{2}, b_{2}\right)=\left(a_{1} \cdot\left(a_{2}\right)^{\varphi\left(b_{1}^{-1}\right)}, b_{1} \cdot b_{2}\right) .
$$

Stated differently, the semidirect product of $A$ and $B$ is the group generated by $A$ and $B$ subject to the relations $a^{b}=a^{\varphi(b)}$ for $a \in A$ and $b \in B$.

2. If $C$ is a group, $\Omega$ a set, and $\psi: B \rightarrow \operatorname{Sym}(\Omega)$ a group homomorphism (that is, an action of $B$ on $\Omega$ ), then we define the wreath product $C \operatorname{wr} B$ of $C$ and $B$ to be the semidirect product of $C^{\Omega}$ and $B$. Here, $C^{\Omega}$ is the group of functions from $\Omega$ to $C$ where group multiplication is defined componentwise, and if we write an element of $C^{\Omega}$ as $\left(c_{i}\right)_{i \in \Omega}$, then the action $\varphi$ of $B$ on $C^{\Omega}$ is given by $\left.\left(\left(c_{i}\right)_{i \in \Omega}\right)^{\varphi(b)}=\left(c_{(i \psi(b-1)}\right)\right)_{i \in \Omega}$.

Now, for $k>0$, define a finitely generated group as follows. Let the symmetric group $S_{3}$ be presented as $\left\langle a, \phi \mid a^{3}=\phi^{2}=a^{\phi} a=1\right\rangle$. Now set

$$
\begin{aligned}
H_{k} & =S_{3} \operatorname{wr} \mathbf{Z}_{2 k+1} \\
& =\left\langle a, \phi, b \mid a^{3}, \phi^{2}, a^{\phi} a, b^{2 k+1},\left[a^{b^{j}}, a^{b^{j^{\prime}}}\right],\left[\phi^{b^{j}}, \phi^{b^{j^{\prime}}}\right],\left[a^{b^{j}}, \phi^{b^{j^{\prime}}}\right]\left(-k \leq j<j^{\prime} \leq k\right)\right\rangle .
\end{aligned}
$$


(Here, the action of $\mathbf{Z}_{2 k+1}$ under consideration is the natural (regular) action of $\mathbf{Z}_{2 k+1}$ on a set of size $2 k+1$.)

Let $L_{k}$ be the subgroup of $H_{k}$ generated by $a, b$, and

$$
t=\prod_{j \in A_{k}} \phi^{b^{j}}
$$

The subgroup $N_{k}$ of the free group $F$ is now the kernel of the canonical homomorphism of $F=\langle a, b, t\rangle$ onto $L_{k}$. (Note here that we have renamed the generators of $F$ to produce a more group-theoretic notation.)

The sequences $\left(H_{k}\right)_{k \in \omega}$ and $\left(L_{k}\right)_{k \in \omega}$ are uniformly computable sequences of finite groups. Namely, $H_{k}$ is just the semidirect product of two groups $C=\bigoplus_{-k<j \leq k} S_{3}$ and $D=\mathbf{Z}_{2 k+1}$, and each element of $H_{k}$ can be uniquely written as $c \cdot d$ with $c \in C$ and $d \in D$ where the multiplication is effective. Thus $\left(N_{k}\right)_{k \in \omega}$ is uniformly computable, and (i) is verified. The best way to visualize $L_{k}$ is as follows: $a$ together with its conjugates by powers of $b$ generates the base of the group, which is of the form $\bigoplus_{-k \leq j \leq k} \mathbf{Z}_{3}, t$ acts componentwise as automorphisms on this base swapping $a^{b^{j}}$ and $\left(a^{2}\right)^{b^{j}}$, if $j$ is in $A_{k}$, and $b$ acts as a shift.

We note the following relations holding in $L_{k}$ (in addition to the ones obviously carrying over from $H_{k}$ ) for all $j, j^{\prime} \in[-k, k]$ :

$$
\begin{gathered}
t^{2}=1, \\
{\left[t^{b^{j}}, t^{b^{j^{\prime}}}\right]=1,} \\
\left(a^{b^{j}}\right)^{t}=\left(a^{b^{j}}\right)^{2}\left(j \in A_{k}\right), \\
\left(a^{b^{j}}\right)^{t}=a^{b^{j}}\left(j \notin A_{k}\right) .
\end{gathered}
$$

Note that $0 \in A_{k} \subseteq[0, k]$ implies in particular that

$$
a^{t}=a^{2} \text { and }\left(a^{b^{j}}\right)^{t}=a^{b^{j}} \text { for all } j \in[-k, 0) .
$$

We say that a word $w$ in $a, b, t$, and their inverses is in normal form if it is of the form

$$
\prod_{i=-d}^{d}\left(a^{t_{i}}\right)^{b^{i}} \cdot \prod_{i=-d}^{d}\left(t^{r_{i}}\right)^{b^{i}} \cdot b^{l},
$$

where $d \in \omega, l \in \mathbf{Z}$, and for $i \in[-d, d], t_{i} \in\{0,1,2\}$ and $r_{i} \in\{0,1\}$.

We begin by showing that

(1) Given a "word" $w \in F$, there is a word $w^{\prime}$ in normal form such that $w=w^{\prime}$ in $L_{k}$ for almost all $k \in \omega$.

(2) If $w$ and $w^{\prime}$ are two distinct words in normal form, then $w \neq w^{\prime}$ in $L_{k}$ for almost all $k \in \omega$.

To show (1), fix a word $w \in F$ and observe first that we can move the $b$ 's in $w$ to the right (at the expense of conjugating by $b$ 's) to obtain a word of the form $w_{0} \cdot b^{l}$, where $w_{0}$ is a product of conjugates of $a$ and $t$ (by powers of $b$ ). Next move all the $a$-conjugates in $w_{0}$ to the left of all the $t$-conjugates using the fact that

$$
t^{b^{s}} a^{b^{r}}= \begin{cases}\left(a^{2}\right)^{b^{r}} t^{b^{s}} & \text { if }(r-s) \in A, \\ a^{b^{r}} t^{b^{s}} & \text { otherwise }\end{cases}
$$


holds in $L_{k}$ for all $r, s$ and all sufficiently large $k$. We thus obtain a word $w_{1} \cdot w_{2} \cdot b^{l}$, where $w_{1}$ is a product of conjugates of $a$ by powers of $b$ and $w_{2}$ is a product of conjugates of $t$ by powers of $b$. Finally use $a^{3}=t^{2}=1$ to obtain the desired $w^{\prime}$ in normal form. $w=w^{\prime}$ holds in $L_{k}$ for $k$ big enough as $A$ is the pointwise limit of the $A_{k}$.

To show (2), fix two distinct words $w=w_{1} \cdot w_{2} \cdot b^{l}$ and $w^{\prime}=w_{1}^{\prime} \cdot w_{2}^{\prime} \cdot b^{l^{\prime}}$ in normal form (where the $w_{1}$ 's and $w_{2}$ 's contain only $a$ 's and $t$ 's, respectively, conjugated by powers of $b$ ). We have to show that $w \neq w^{\prime}$ holds in almost all $L_{k}$ or equivalently in almost all $H_{k}$.

Remember that $H_{k}$ is the semidirect product of $C=\bigoplus_{-k \leq i \leq k} S_{3}$ and $\mathbf{Z}_{2 k+1}$. We can write each of the involved groups $S_{3}$ as a semidirect product $\mathbf{Z}_{3}$ and $\mathbf{Z}_{2}$, where $\mathbf{Z}_{3}$ is generated by $a^{b^{j}}$ for an appropriate $j$. So we can also write $C$ as a semidirect product of $\bigoplus_{-k \leq i \leq k} \mathbf{Z}_{3}$ and $\bigoplus_{-k \leq i \leq k} \mathbf{Z}_{2} . w_{1}$ is a product of conjugates of $a$ and therefore always belongs to the first group, while $w_{2}$ belongs to the second group and $b^{l}$ belongs to $\mathbf{Z}_{2 k+1}$. This implies that $w_{1} \cdot w_{2} \cdot b^{l}=w_{1}^{\prime} \cdot w_{2}^{\prime} \cdot b^{l^{\prime}}$ holds in $H_{k}$ iff $w_{1}=w_{1}^{\prime}$ and $w_{2}=w_{2}^{\prime}$ and $b^{l}=b^{l^{\prime}}$ holds in $H_{k}$. Therefore, if the words $w_{1}$ and $w_{1}^{\prime}$, or the words $b^{l}$ and $b^{l^{\prime}}$, are distinct, then obviously $w_{1} \cdot w_{2} \cdot b^{l} \neq w_{1}^{\prime} \cdot w_{2}^{\prime} \cdot b^{l^{\prime}}$ in almost all $H_{k}$.

So assume $l=l^{\prime}$ and $w_{1}=w_{1}^{\prime}$ from now on, and we simply have to show that $w_{2} \neq w_{2}^{\prime}$ in $F$ implies $w_{2} \neq w_{2}^{\prime}$ in almost all $H_{k}$ 's. For this, it suffices to show that any nontrivial word of the form

$$
v=\prod_{i=e}^{f}\left(t^{r_{i}}\right)^{b^{i}}
$$

does not equal 1 in almost all $H_{k}$ 's (where $e \leq f$ are integers, $r_{i} \in\{0,1\}$ for $i \in[e, f]$, and $\left.r_{e}=r_{f}=1\right)$. Now note that by $(* * *)$ above, for $k \geq f-e$ the following holds in $H_{k}$ :

$$
\prod_{i=e}^{f}\left(t^{r_{i}}\right)^{b^{i}} \cdot a^{b^{e}}=t^{b^{e}} \cdot a^{b^{e}} \cdot \prod_{i=e+1}^{f}\left(t^{r_{i}}\right)^{b^{i}}=\left(a^{2}\right)^{b^{e}} \cdot \prod_{i=e}^{f}\left(t^{r_{i}}\right)^{b^{i}} .
$$

But this means that for $k \geq f-e,\left(a^{b^{e}}\right)^{v}=\left(a^{b^{e}}\right)^{2}$ which implies $v \neq 1$ as $a \neq a^{2}$ in $H_{k}$.

We can now verify the remaining claims of the lemma as follows:

(ii) holds by (1) and (2) above.

(iii) holds since $x \in A$ iff $t a^{b^{x}} t a^{b^{x}} \in N=\lim _{k} N_{k}$ (see $\left(^{*}\right)$ and $\left.(* *)\right)$ and as the process of getting a word in normal form is computable in $A$.

This concludes the proof of the lemma.

Given the lemma, we can now easily finish the proof of the theorem. For each $k \in \omega$, define the Cayley graph $\mathcal{C}_{k}$ of $F / N_{k}$ by setting the universe $C_{k}=F / N_{k}$ and defining three binary relations $R_{0}, R_{1}$, and $R_{2}$ on $C_{k}$ by setting $R_{i}(v, w)$ iff $v=w g_{i}$ in $F / N_{k}$ (for $i \leq 2$ ). Similarly, we define the Cayley graph $\mathcal{C}$ of $F / N$. We then define $\mathcal{M}_{0}$ as the disjoint union of the Cayley graphs $\mathcal{C}_{k}$ (for all $k \in \omega$ ). For any cardinal $\kappa>0$, we define $\mathcal{M}_{\kappa}$ to be the disjoint union of $\mathcal{M}_{0}$ and $\kappa$ many copies of the Cayley graph $\mathcal{C}$. Let $c \in C$.

By (ii) of the group-theoretical lemma, for any fixed $r>0, B_{r}(x)$ is isomorphic to $B_{r}(c)$ for almost all $x \in \mathcal{M}_{0}$. Thus by the lemma in Section $1, \mathcal{M}_{0}$ is strongly minimal, the other models of the theory being the $M_{\kappa}$. 
This establishes (i) of the theorem. It is clear from the above construction and (i) and (iii) of the group-theoretical lemma, respectively, that for $\kappa \leq \omega, \mathcal{M}_{\kappa}$ is constructive iff $\kappa=0$.

We finish the proof by showing how $A$ is computable from any representation of $\mathcal{M}_{\kappa}$ for $1 \leq \kappa \leq \omega$. (Recall that by an observation of Knight [Kn86], the set of degrees of presentations of a countably infinite model is upward closed iff its automorphism group does not contain the stabilizer (in the symmetric group) of a finite subset of the model.) Pick $c \in \mathcal{M}_{\kappa}-\mathcal{M}_{0}$. Let $x \in \omega$. To decide if $x \in A$, compute $B_{2 k+3}(x)$ (that is effective in the given presentation of $\mathcal{M}_{\kappa}$ ), which is part of the Cayley graph of $F / N$ and decide if $t a^{b^{x}}=\left(a^{2}\right)^{b^{x}} t$.

\section{CONCLUding REMARKS}

We remark that our theorem leaves open the question of exactly which subsets $S$ of $\omega+1$ can be realized as spectra of constructive models of uncountably categorical theories. In fact, it is even unclear whether all such sets $S$ must be arithmetical.

Closely related to this is the question of how complicated the other countable models of an uncountably categorical theory of a constructive model can be. By an observation (jointly with T. Millar), if the language $\mathcal{L}$ is finite and contains only binary relation symbols, and the prime model $\mathcal{M}_{0}$ is constructive and strongly minimal, then the other countable models of $\operatorname{Th}\left(\mathcal{M}_{0}\right)$ must have presentations computable in $\mathbf{0}^{\prime \prime}$, the second Turing jump of the computable Turing degree. Since the geometry of models in relations of higher arity can be much more complicated, the situation is unclear in this case and appears quite hard.

If we content ourselves with an uncountably categorical theory as opposed to a strongly minimal theory, then we can find a theory of graphs satisfying the three conditions of our theorem. In fact this is true for all the questions of similar type, e.g. for the ones posed above in this section: We could as well restrict our attention to graphs.

This is true because standard techniques in model theory allow one to interpret any given structure of finite signature in a graph in a way that respects features like categoricity or computability (but not strong minimality). For an example of this technique see, e.g., chapter 10.3 in Ebbinghaus, Flum [EF95]. In fact the interpretation can be chosen to be a bi-interpretation in the sense of Ahlbrandt, Ziegler [AZ86]. In the present context we need an interpretation which is effective in both directions. By an effective interpretation of one countable structure in another we mean that the domain and relations of the first structure are computable in any presentation of the second structure. In particular, in the case of an effective biinterpretation, any presentation of one structure leads to a presentation of the second structure of the same Turing degree.

Let us sketch how this can be done:

Proposition. Let $\mathcal{M}$ be a countable structure in a finite language. There exists a graph $\mathcal{N}$ which is bi-interpretable with $\mathcal{M}$; both interpretations are effective.

Proof. We can easily suppose that the language of the original structure $\mathcal{M}$ is relational and that the unary predicates give a partition of the domain of $\mathcal{M}$. First we introduce a new point $p_{R, \bar{a}}$ for every $n$-ary relation symbol $R$ (for $n>1$ ) and for every $n$-tuple $\bar{a}$ from $\mathcal{M}$. The domain of the new structure $\mathcal{M}_{1}$ now consists of all the points from $\mathcal{M}$ and the new points. Let $V$ be the set of points from $\mathcal{M}$. We replace every $n$-ary symbol $R$ of the old language by unary predicates 
$Y_{R}$ and $N_{R}$. The interpretation of $Y_{R}$ in $\mathcal{M}_{1}$ is $\left\{p_{R, \bar{a}} \mid \mathcal{M}=R \bar{a}\right\}$ and of $N_{R}$ is $\left\{p_{R, \bar{a}} \mid \mathcal{M} \models \neg R \bar{a}\right\}$ ( $Y$ and $N$ for Yes and No). We keep the unary predicates already present in $\mathcal{M}$ and their interpretations. Moreover, if $n$ is the maximal arity of the old language, we introduce $n$ many binary symbols $\left\{T_{j}\right\}_{0 \leq j<n}$ and $T_{j}$ is interpreted by the relation $\left\{\left(b, p_{R, \bar{a}}\right) \mid b\right.$ is the $j$-th component of the tuple $\left.\bar{a}\right\}$. We can interpret $\mathcal{M}$ in $\mathcal{M}_{1}$ in a computable fashion. E.g., $R x_{0} \ldots x_{n-1}$ can be defined by the formula $\exists y\left(Y_{R} y \wedge T_{0} x_{0} y \wedge \ldots \wedge T_{n-1} x_{n-1} y\right)$, which is computable in the structure $\mathcal{M}_{1}$ as for every $x_{0}, \ldots, x_{n-1}$ exactly one of $\exists y\left(Y_{R} y \wedge T_{0} x_{0} y \wedge \ldots \wedge T_{n-1} x_{n-1} y\right)$ and $\exists y\left(N_{R} y \wedge T_{0} x_{0} y \wedge \ldots \wedge T_{n-1} x_{n-1} y\right)$ holds. We can consider the structure $\mathcal{M}_{1}$ as a directed colored graph, where the edges and the vertices are colored. Furthermore, every edge connects a point belonging to $V$ to one not belonging to $V$.

Now we apply the same procedure to $\mathcal{M}_{1}$, except that we only introduce new points $p_{S,(a, b)}$ for each binary relation $S$ in the language of $\mathcal{M}_{1}$ and each $a \in V$ and $b \notin V$. This suffices, as only between such points can a relation hold in $\mathcal{M}_{1}$. Furthermore, we can replace the only two relation symbols $T_{0}$ and $T_{1}$ of the new structure by the symbol $T$ with the defining clause $T x y \leftrightarrow\left(T_{0} x y \vee T_{1} x y \vee T_{0} y x \vee\right.$ $\left.T_{1} y x\right)$ without losing any information. We call the resulting structure $\mathcal{M}_{2} . \mathcal{M}_{2}$ is a colored graph (where only the vertices are colored), which is bipartite, and which does not have any isolated points.

Let $\left\{U_{1}, \ldots, U_{r}\right\}$ be the unary predicates in the language of $\mathcal{M}_{2}$. In the final step, for every point $a$ of $\mathcal{M}_{2}$ if $\mathcal{M}_{2} \models U_{j} a$, we introduce $2 j$ many new points $a_{1}, \ldots, a_{2 j}$ and we enlarge the interpretation of $T$ by the pairs $\left(a_{i}, a_{j}\right)$, where $(i-j) \equiv 1 \bmod$ $2 j+1$ or $(i-j) \equiv-1 \bmod 2 j+1\left(\right.$ where $\left.a_{0}=a\right)$. In other words, we add a loop of size $2 j+1$. Let $\mathcal{N}$ be the resulting $\{T\}$-structure. $\mathcal{N}$ is a graph, and it is easy to see that $\mathcal{M}_{2}$ is interpretable in an effective way in $\mathcal{N}$. The clue is that $\mathcal{M}_{2}$ is bi-partite, so there are no odd cycles in $\mathcal{M}_{2}$. The newly introduced loops are the only odd cycles in $\mathcal{N}$ without repetition. Every point of $\mathcal{N}$ belongs to exactly one such cycle as the unary predicates of $\mathcal{M}_{2}$ give a partition of $\mathcal{M}_{2}$. In every such cycle there is exactly one point with more than two neighbors.

Now $\mathcal{M}$ and $\mathcal{N}$ are bi-interpretable, and the interpretations are effective.

\section{REFERENCES}

[AZ86] Ahlbrandt, G. and Ziegler, M., Quasi-finitely axiomatizable totally categorical theories, Stability in model theory (Trento, 1984), Ann. Pure Appl. Logic 30 (1986), 63-82. MR 87k:03026

[BL71] Baldwin, J. T. and Lachlan, A. H., On strongly minimal sets, J. Symbolic Logic 36 (1971), 79-96. MR 44:3851

[EF95] Ebbinghaus, H.-D. and Flum, J., Finite model theory, Perspectives in Mathematical Logic, Springer-Verlag, Berlin, 1995. CMP 97:01

[Go78] Gončarov, S. S., Constructive models of $\aleph_{1}$-categorical theories, Mat. Zametki 23 (1978), 885-888 (Russian). MR 80g:03029

[Ha74] Harrington, L., Recursively presented prime models, J. Symbolic Logic 39 (1974), 305309. MR 50:4292

[Hi74] Hisamiev, N. G., On strongly constructive models of decidable theories, Izv. Akad. Nauk Kazakh. SSR Ser. Fiz.-Mat. 35 (1) (1974), 83-84 (Russian). MR 50:6824

[Iv89] Ivanov, A. A., The problem of finite axiomatizability for strongly minimal theories of graphs, and groups with more than one end, Algebra i Logika 28 (1989), 160-173 (Russian). MR 91m:03035

[Iv89b] Ivanov, A. A., The problem of finite axiomatizability for strongly minimal theories of graphs, Algebra i Logika 28 (1989), 280-297 (Russian). MR 91i:03069

[KNSta] Khoussainov, B., Nies, A., and Shore, R. A., Recursive models of theories with few models (to appear). 
[Kn86] Knight, J. F., Degrees coded in jumps of orderings, J. Symbolic Logic 51 (1986), 10341042. MR 88j:03030

[Ku80] Kudaĭbergenov, K. Ž., Constructivizable models of undecidable theories, Sibirsk. Mat. Zh. 21 (5) (1980), 155-158, 192. MR 82h:03040

[Mo65] Morley, M., Categoricity in power, Trans. Amer. Math. Soc. 114 (1965), 514-538. MR 31:58

[Ro95] Rotman, J., An introduction to the theory of groups, 4th edition, Graduate Texts in Mathematics No. 148, Springer-Verlag, New York, 1995. MR 95m:20001

School of Mathematics, University of Leeds, LeEds LS2 9JT, England

E-mail address: herwig@amsta.leeds.ac.uk

Current address: Institut für Mathematische Logik, Albert-Ludwigs-Universität Freiburg, D79104 Freiburg, Germany

E-mail address: herwig@ruf.uni-freiburg.de

Department of Mathematics, University of Wisconsin, Madison, Wisconsin 53706-1388

E-mail address: lempp@math.wisc.edu

Institut für Mathematische Logik, Albert-Ludwigs-Universität Freiburg, D-79104 Freiburg, Germany

E-mail address: ziegler@uni-freiburg.de 